Regular Article

\title{
Selective impact of Tau loss on nociceptive primary afferents and pain sensation
}

\author{
Ioannis Sotiropoulos ${ }^{\mathrm{a}, \mathrm{b}}$, André T. Lopes ${ }^{\mathrm{a}, \mathrm{b}}$, Vitor Pinto ${ }^{\mathrm{a}, \mathrm{b}}$, Sofia Lopes $^{\mathrm{a}, \mathrm{b}}$, Sara Carlos ${ }^{\mathrm{a}, \mathrm{b}}$, Sara Duarte-Silva ${ }^{\mathrm{a}, \mathrm{b}}$, \\ Andreia Neves-Carvalho ${ }^{\mathrm{a}, \mathrm{b}}$, Filipa Pinto-Ribeiro ${ }^{\mathrm{a}, \mathrm{b}}$, Sara Pinheiro ${ }^{\mathrm{a}, \mathrm{b}}$, Rui Fernandes ${ }^{\mathrm{c}}$, Armando Almeida ${ }^{\mathrm{a}, \mathrm{b}}$, \\ Nuno Sousa ${ }^{\mathrm{a}, \mathrm{b}, *}$, Hugo Leite-Almeida ${ }^{\mathrm{a}, \mathrm{b}, *}$
}

a Life and Health Sciences Research Institute (ICVS), School of Health Sciences, University of Minho, Campus Gualtar, 4710-057 Braga, Portugal

b ICVS/3B's - PT Government Associate Laboratory, Braga/Guimarães, Portugal

c TEM unit, IBMC, Porto, Portugal

\section{A R T I C L E I N F O}

\section{Article history:}

Received 19 March 2014

Revised 8 July 2014

Accepted 20 July 2014

Available online 28 July 2014

\section{Keywords:}

Tau

Pain

Peripheral nerve

Myelination

C-fibers

\begin{abstract}
A B S T R A C T
Tau protein hyperphosphorylation and consequent malfunction are hallmarks of Alzheimer's disease pathology; importantly, pain perception is diminished in these patients. In physiological conditions, Tau contributes to cytoskeletal dynamics and in this way, influences a number of cellular mechanisms including axonal trafficking, myelination and synaptic plasticity, processes that are also implicated in pain perception. However, there is no in vivo evidence clarifying the role of Tau in nociception. Thus, we tested Tau-null (Tau -/ - ) and Tau $+/+$ mice for acute thermal pain (Hargreaves' test), acute and tonic inflammatory pain (formalin test) and mechanical allodynia (Von Frey test). We report that Tau $-/-$ animals presented a decreased response to acute noxious stimuli when compared to Tau $+/+$ while their pain-related behavior is augmented under tonic painful stimuli. This increased reactivity to tonic pain was accompanied by enhanced formalin-evoked c-fos staining of second order nociceptive neurons at Tau-null dorsal horn. In addition, we analyzed the primary afferents conveying nociceptive signals, estimating sciatic nerve fiber density, myelination and nerve conduction. Ultrastructural analysis revealed a decreased C-fiber density in the sciatic nerve of Tau-null mice and a hypomyelination of myelinated fibers (Aס-fibers) - also confirmed by western blot analysis - followed by altered conduction properties of Tau-null sciatic nerves. To our knowledge, this is the first in vivo study that demonstrates that Tau depletion negatively affects the main systems conveying nociceptive information to the CNS, adding to our knowledge about Tau function(s) that might also be relevant for understanding peripheral neurological deficits in different Tauopathies.
\end{abstract}

(c) 2014 Elsevier Inc. All rights reserved.

\section{Introduction}

Tau malfunction, through its abnormal hyperphosphorylation, is postulated as a crucial mechanism of Alzheimer's disease (AD) neuronal dysfunction but it remains unknown whether Tau protein could be a direct or indirect contributor to altered pain processing found in $\mathrm{AD}$ patients (Corbett et al., 2012). The predominant view about Tau function focuses on its key cytoskeletal role based on its ability to bind to microtubules (MT) and other cytoskeletal elements (Mandelkow and Mandelkow, 2012). Interestingly, neuropathic pain is a common side effect of MTtargeting agents commonly used in the clinics as chemotherapeutic

Abbreviations: AD, Alzheimer's disease; NMDA, N-methyl-D-aspartate; CNS, central nervous system; PNS, peripheral nervous system; MT, microtubules; MBP, myelin basic protein.

* Corresponding authors at: ICVS, Life and Health Sciences School, University of Minho, 4710-057 Braga, Portugal.

E-mail addresses: njcsousa@ecsaude.uminho.pt (N. Sousa), hugoalmeida@ecsaude.uminho.pt (H. Leite-Almeida). drugs (e.g. vincristine, paclitaxel) (Jaggi and Singh, 2012). In addition, a growing body of evidence suggests that Tau has an important role in the dendritic compartment where, via interaction with Fyn, contributes to NMDA receptor/PSD95 complex formation (Frandemiche et al., 2014; Ittner and Gotz, 2011) that has an established role in painrelated sensitization (D'Mello et al., 2011). There is also direct evidence of Fyn involvement on pain sensitization through phosphorylation of the NMDA NR2B subunit (Abe et al., 2005). Furthermore, Fyn constitutional activation results in hyperalgesia in naive mice (Liu et al., 2014). Moreover, a cell culture-based study suggests that Tau-Fyn interaction may be involved in myelination regulation (Klein et al., 2002), which is of relevance for optimal axonal function and, consequently, for nociceptive transmission. Furthermore, there is also evidence that other cytoskeletal elements may be involved in pain as disruptors of microtubules, actin filaments and neurofilaments in primary afferent nociceptors interfere with normal pain sensation (Reichling and Levine, 2011) reinforcing the importance of understanding the relation between cytoskeletal proteins and pain. 
An initial step for pain perception is the activation of peripheral nociceptors which generates a local depolarization conveyed by specific fibers to secondary afferent neurons in the dorsal horn. Responding to mechanical, thermal and/or chemical stimuli, A $\delta$ fibers are the smalldiameter myelinated fibers that participate in the transmission of noxious stimuli together with the unmyelinated C-fibers. Myelination problems as well as reduced number of fibers in sciatic nerve have been shown to affect pain perception and pain circuitry function (Chen et al., 2003). While previous findings described alterations in small-caliber axons of animals lacking Tau protein (Harada et al., 1994), and despite the suggestion that Tau protein is implicated in myelination processes (Klein et al., 2002), yet, surprisingly, to the best of our knowledge, there is no in vivo evidence clarifying the role of Tau in nociception. Thus, herein, we assessed pain behavior in animals lacking Tau followed by ultrastructure, molecular and electrophysiological analysis of their sciatic nerve.

\section{Materials and methods}

\section{Animals}

This study used 4-6 month-old male Tau-null (Tau-/-) and Tau +/+ (C57BL/6) animals (Dawson et al., 2001). Adult Tau-null animals of this strain exhibited no cognitive or locomotor deficits as previously described (Dawson et al., 2010; Gotz et al., 2013). All animals were used in accordance with European Union Council Directive 86/609/EEC and local welfare regulations. Mice were housed in groups of 4 to 5 animals per cage under standard environmental conditions (ambient temperature of $21 \pm 1{ }^{\circ} \mathrm{C}$ and a relative humidity of 50-60\%; $12 \mathrm{~h} \mathrm{light/dark}$ cycle (lights on at 8:00 A.M.) ad libitum access to food and water).

\section{Pain-related behaviors}

\section{Hargreaves' test (acute thermal noxious stimulus)}

Animals ( $\mathrm{N}=18-20$ per genotype) were submitted to Hargreaves' test which consists of a radiant heat applied to the plantar skin of the hind limb and withdrawal latency was automatically registered using radiant heat equipment (Plantar Test Device Model 7370, Ugo Basile, Comerio, Italy) as previously described (Pinto-Ribeiro et al., 2013). After acclimatization period, the measurement was repeated 4 times with 60 second intervals. Cut-off time was $10 \mathrm{~s}$. The experimenter was blind to the animals' genotype.

\section{Formalin test (inflammatory stimulus)}

The formalin test was performed as previously described (Almeida et al., 1999). Briefly, $50 \mu \mathrm{L}$ of $5 \%$ formalin was injected subcutaneously in the dorsal surface of the right hind-paw. Animals were then placed over a transparent acrylic surface within a $10 \mathrm{~cm}^{3}$ transparent acrylic box with perforations in all walls for proper air ventilation and sufficient space for animals to freely move. Animals were filmed from below for $60 \mathrm{~min}$ and pain-related behaviors (paw protection/licking periods and paw jerks) in the acute (0-5 min) and tonic (15-60 min) phases were posteriorly quantified by two independent observers using EthoLog software (Ottoni, 2000). Prior to the experiment, animals were acclimatized to the experimental conditions to minimize potential stress effects. Experimental groups for Tau-null and Tau $+/+$ were divided in: saline-injected (control) animals (Sal; $N=10$ ) and formalin-injected animals (Form; $\mathrm{N}=13-15$ ). The experimenter was blind to the animals' genotype and drug.

\section{Von Frey test (up-and-down method)}

Tactile allodynia (pain evoked by a normally innocuous stimulus) was accessed by the up-and-down method (Chaplan et al., 1994). Animals (10-11 animals per group) were placed in an elevated grid and the glabrous skin of the hind paw was probed with a series of Von Frey calibrated monofilaments: $2.0 \mathrm{~g}, 1.0 \mathrm{~g}, 0.6 \mathrm{~g}, 0.4 \mathrm{~g}, 0.16 \mathrm{~g}, 0.07 \mathrm{~g}$, and $0.04 \mathrm{~g}$ (North Coast Medical Inc., USA). Starting with the $0.4 \mathrm{~g}$ filament, the test would advance upward if no response was elicited $(=0)$ or downward if a brisk withdrawn of the limb was produced $(=\mathrm{X})$ until 6 measurements were obtained around the threshold point according to the model developed by Dixon (1980). The 50\% response threshold was then calculated using the formula $50 \% \mathrm{~g}$ threshold $=\left(10^{\wedge}\left[\mathrm{X}_{\mathrm{f}}+\mathrm{K} . \delta\right]\right) / 10.000$, where $\mathrm{X}_{\mathrm{f}}=$ value (in log units) of the final Von Frey filament; $\mathrm{k}=$ tabular value corresponding to pattern of positive and negative responses ( $\mathrm{X}$ and $\mathrm{O}$ sequences; consult (Chaplan et al., 1994)); $\delta=$ mean difference (in log units) between stimuli (0.267). If no response $(=0)$ was obtained up to maximal force $(2.0 \mathrm{~g})$ or conversely, if all filaments elicited a response $(=\mathrm{X})$ down to the minimal force $(0.04 \mathrm{~g})$, these values were assumed as the $50 \%$ withdrawal threshold. The experimenter was blind to the animals' genotype while animals were habituated to the experimental conditions for 2 days before the experiment to minimize potential stress effects.

\section{c-fos immunohistochemistry and density estimation}

90 min after formalin test completion, animals were deeply anesthetized with sodium pentobarbital (CEVA Saúde Animal, Portugal) and then perfused through the ascending aorta with PBS followed with $4 \%$ paraformaldehyde in PBS. The spinal cord was removed and immersed in the same fixative for $24 \mathrm{~h}$ at room temperature (RT) and then, moved to an $8 \%$ sucrose solution and kept at $4{ }^{\circ} \mathrm{C}$ until use. $50 \mu \mathrm{m}$ coronal sections of the lumbar area were then obtained in a vibratome (Leica 1000VTS) and serially collected in PBS. For c-fos IHC, sections were incubated in 3.3\% $\mathrm{H}_{2} \mathrm{O}_{2}$ (in PBS, pH 7.2) for $30 \mathrm{~min}$, RT and then, after several washes in PBS and 0.3\% PBS-T (Triton-X-100; Sigma), incubated in $2.5 \%$ FBS (fetal bovine serum; Stem Cell, 062-00) in PBS-T 2 hour RT, followed by a solution of 1:2000 anti-Fos (Calbiochem, PC38-100) and 2.0\% FBS in PBS-T overnight at RT. Sections were then washed several times in PBS$\mathrm{T}$ and incubated for $1 \mathrm{~h}$, RT in a solution of 1:200 biotinylated swine anti-rabbit secondary antibody (Dako, E0353). Sections were then washed in PBS-T and incubated for $1 \mathrm{~h}, \mathrm{RT}$ in a solution of 1:200 avidin-biotin complex (ABC, Vector Laboratories). Sections were again washed with PBS-T, PBS and Tris-HCL $(0.05 \mathrm{M}, \mathrm{pH} 7.6)$ and briefly incubated in $0.0005 \%(\mathrm{w} / \mathrm{v})$ diaminobenzidine tetrahydrochloride (DAB; Sigma Immunochemicals, St. Louis, USA), $0.0002 \% \mathrm{H}_{2} \mathrm{O}_{2}$ (v/v) in Tris$\mathrm{HCl}$. Sections were then serially placed in Superfrost Plus slides (Menzel-Gläser, Braunschweig, Germany), dehydrated and mounted in Entellan (Merck, Darmstadt, Germany). An Olympus BX51 microscope equipped with U-MAD3 camera was used to acquire images from the left and right dorsal horn of spinal cord. c-fos density was manually determined in specific outer (laminae I-II) and deeper (laminae III-V) regions by an experimenter (AL) blind to the samples provenience (i.e. saline or formalin of both genotypes; 5-6 animals per group).

\section{Sciatic nerve ultrastructure analysis}

Sciatic nerves of undisturbed Tau-null and Tau $+/+$ animals were collected and fixed in $4 \%$ glutaraldehyde in $0.1 \mathrm{M}$ sodium cacodylate buffer $\mathrm{pH} 7.4$ for 1 week. Then, they were postfixed in $1 \% \mathrm{OsO}_{4}$, dehydrated and embedded in epon (Electron Microscopy Sciences). $1 \mu \mathrm{m}$ thick transverse sections covering the complete cross-sectional nerve area were stained with $1 \% \mathrm{p}$-phenylene diamine (PPD) for 10 min and mounted on Entellan (Merck). An Olympus DP70 was used to photograph the transverse sections of the nerve and images were mounted on Photoshop. For each animal ( $\mathrm{N}=5-6$ per genotype), the total number of myelinated fibers in one transverse section was counted. The g-ratio [axon diameter / (axon diameter + myelin thickness)] was determined through the analysis of at least 100 fibers. For electron microscopy, ultrathin-sections (60 $\mathrm{nm}$ ) were counterstained with alcoholic uranyl acetate solution, followed by aqueous uranyl acetate solution and lead citrate. Grids were observed on a JEOL JEM-1400 transmission electron microscope equipped with an Orius Sc1000 digital camera. 16 non-overlapping images were obtained and used for all 
determinations (number axons/bundle and density of unmyelinated fibers) by an experimenter in a manual way; experimenter was blind to the samples' provenience (Tau-null and Tau+/+; 5 animals per genotype).

\section{Ex-vivo measurement of compound action potentials}

Acutely isolated sciatic nerves from Tau-null and Tau $+/+$ animals (5-6 animals per group) were used for assessment of A- and C-fiber conduction velocity and compound action potential (CAP) area as previously described (Pinto et al., 2008). Briefly, sciatic nerves were dissected and cleaned from the connective tissue sheath in artificial cerebrospinal fluid (ACSF) containing (in $\mathrm{mM}$ ): $124 \mathrm{NaCl}, 2.5 \mathrm{KCl}, 1 \mathrm{MgSO}_{4}, 2 \mathrm{CaCl}_{2}$, $1.25 \mathrm{NaH}_{2} \mathrm{PO}_{4}, 26 \mathrm{NaHCO}_{3}$, and 10 Glucose, bubbled with carbogen gas $\left(95 \% \mathrm{O}_{2}, 5 \% \mathrm{CO}_{2}\right)$. After isolation, the nerves were used at room temperature of $22-24{ }^{\circ} \mathrm{C}$. CAP recordings were made with a Multiclamp 700B amplifier in VC mode and digitized with the Digidata 1440A digitizer using pClamp 10 software (Axon Instruments). Signals were low-pass filtered at an effective corner frequency of $16 \mathrm{kHz}$ and sampled at $50 \mathrm{kHz}$. Fibers were stimulated through suction electrodes using an isolated pulse stimulator (STG4002, multichannel systems). Short pulses of $50 \mu$ s were applied to activate CAPs in A $\alpha \beta$ - and A $\delta$-fibers. C-fibers were stimulated with $1 \mathrm{~ms}$ current pulses. Sciatic nerves were repeatedly stimulated with current pulses of increasing amplitudes until saturation. Conduction velocities were calculated for the CAP peak of Afibers and for the first peak of the C-fiber biphasic CAP. Total areas were calculated using clampfit software (Axon Instruments). Electrophysiological recordings and analyses were performed by an experimenter blind to the provenience of the tissue.

\section{Western blot analysis}

Sciatic nerves of undisturbed Tau-null and Tau $+/+$ animals (6-7 animals per genotype) were isolated and homogenized in lysis buffer [10 mM HEPES pH 7.9, 150 mM NaCl, 1 mM EGTA, 1 mM EDTA, 10\% glycerol, 1\% NP-40, Complete Protease Inhibitor (Roche, Mannheim, Germany) and Phosphatase Inhibitor Cocktails II and III (Sigma, St Louis, MO)] using a Dounce glass homogenizer; extracts were sonicated and centrifuged $\left(15.000 \mathrm{~g} ; 10 \mathrm{~min} ; 4^{\circ} \mathrm{C}\right.$ ) and their protein contents were estimated (Bradford assay). After reconstitution in Laemmli buffer (250 mM Tris- $\mathrm{HCl}$, pH 6.8, containing 4\% sodium dodecyl sulfate, $10 \%$ glycerol, $2 \%$ b-mercaptoethanol and $0.002 \%$ bromophenol blue), $25 \mu \mathrm{g}$ of lysates was electrophoresed on $10 \%$ acrylamide gels, and transferred onto nitrocellulose membranes (BIORAD Turbo) while transfer efficacy was checked by membrane Ponceau staining. Membranes were blocked in Tris-buffered saline containing 5\% non-fat milk and 0.2\% Tween-20 before incubation with myelin basic protein (MBP) antibody (Serotec; 1:500) and actin (DSHB 1:2000). Antigens were revealed by enhanced chemiluminescence (BIORAD) after incubation with appropriate horseradish peroxidase-immunoglobulin $\mathrm{G}$ conjugates (BIORAD). Protein ladder (Fermentas) was used to monitor molecular weight of proteins of interest. Blots were scanned and quantified using TINA 3.0 bioimaging software (Raytest, Germany). All values were normalized against actin and expressed as percentages of control.

\section{Statistical analysis}

All data sets were subjected to t-test analysis except c-fos expression data that were analyzed by 2-way ANOVA having genotype (Tau $+/+$ vs Tau $-/-$ ) and treatment (saline vs formalin) as factors and it was followed by Tukey post-hoc analysis (SPSS, Aspire Software, USA). Differences were considered to be significant if $\mathrm{p}<0.05$.

\section{Results}

Tau-null animals present decreased nociception to acute noxious but the opposite to tonic stimulation

For detailed characterization of Tau involvement in nociception, Tau-null and Tau+/+ (wild-type) animals were submitted to different pain-related behavioral tests that are used to examine relevant painlike responses. The response latency to acute noxious heat $\left(54{ }^{\circ} \mathrm{C}\right)$ on the hind paw (Hargreaves' test) was significantly increased in Taunull when compared to wild type animals $(t=2.865, p<0.05)$ indicating reduced nociception to acute noxious stimuli (Fig. 1A). Furthermore, we evaluated inflammatory pain in both genotypes using the formalin test. Pain-related behaviors such as protecting/licking and paw jerks were monitored for $1 \mathrm{~h}$ after subcutaneous paw injection of formalin in the right hind paw (Figs. 1B and C). In line with the above test of acute pain, the acute phase of the formalin test $(0-5 \mathrm{~min})$ shows that Tau-null animals spent less time engaged in pain-related behaviors as indicated by the time of protecting/licking the formalin-injected paw $(\mathrm{t}=2.162, \mathrm{p}<0.05$; Fig. 1B left). In addition, Tau-null animals displayed decreased number of jerks of the formalin-injected paw when compared to Tau $+/+(t=2.933, p<0.05$; Fig. 1C left $)$; both typical pain-related behaviors induced by formalin suggest that Tau-null animals respond less to the acute phase of formalin noxious stimulation. Interestingly, animals from the two genotypes exhibit a different response profile in the tonic phase of the formalin test (15-60 min). Specifically, an increased protecting/licking behavior was found in Tau-null animals when compared to Tau $+/+$ animals $(t=2.089, \mathrm{p}<0.05$; Fig. 1B right) indicating increased pain, though no significant difference in the number of paw jerks (Fig. 1C right). Importantly, Tau-null and Tau $+/+$ animals presented no differences in their response threshold to innocuous light mechanical stimulus (Fig. 1D) suggesting that normal tactile sensation is preserved while the observed alterations phenotypes were restricted to pain.

Spinal cord formalin-induced c-fos expression is higher in Tau-null animals

c-fos positive cell density is used as a central correlate of pain-related neuronal activity driven by formalin stimulation of peripheral afferents (Fig. 2A). Thus, we measured c-fos-positive neurons in lumbar L4-L6 spinal cord of the formalin- and saline-injected animals. 2-Way ANOVA analysis showed that c-fos cell density in ipsilateral and contralateral I-II (superficial) and III-IV (deep) spinal cord lamina was significantly dependent on the treatment (formalin vs saline: $\mathrm{ipsi}_{\mathrm{I}-\mathrm{II}} \mathrm{F}_{1,21}=29.039$; contra $_{\text {III }} \mathrm{F}_{1,21}=42.714$; ipsi $\mathrm{iII-V}_{\mathrm{V}} \mathrm{F}_{1,21}=31.321$; contra ${ }_{\text {III-V }} \mathrm{F}_{1,21}=$ 22.159; $\mathrm{p}<0.001$ in all cases) and genotype (Tau-null vs Tau $+/+$ : ipsi $_{\text {I-II }} \mathrm{F}_{1,21}=4.560, \mathrm{p}=0.047$; contra $_{\mathrm{I}-\mathrm{II}} \mathrm{F}_{1,21}=25.746, \mathrm{p}<0.001$; ipsi $_{\text {III-V }} \mathrm{F}_{1,21}=5.372, \mathrm{p}=0.032$; contra $\mathrm{III}_{\mathrm{V}} \mathrm{F}_{1,21}=9.016, \mathrm{p}=0.008$ ). Also, the interaction of these factors resulted significantly in almost all comparisons - ipsi $\mathrm{I}_{\mathrm{III}} \mathrm{F}_{1,21}=4.098, \mathrm{p}=0.058$; contra $_{\mathrm{I}-\mathrm{II}} \mathrm{F}_{1,21}=$ 20.892, $\mathrm{p}<0.001$; ipsi $\mathrm{i}_{\text {III-V }} \mathrm{F}_{1,21}=5.343, \mathrm{p}=0.033$; contra III-V $\mathrm{F}_{1,21}=$ $6.490, p=0.020$. As shown in Fig. $2 \mathrm{~B}$, formalin injection resulted in an expected increased c-fos + density in the ipsilateral dorsal horn. However, this effect had a higher magnitude in Tau-null animals matching with their increased pain-related behavioral responses at the tonic phase of the formalin test. Interestingly, Tau-null animals demonstrated an abnormal c-fos staining at both layers I-II and III-V of the contralateral side of spinal cord as shown in Fig. 2A.

Tau-null sciatic nerves exhibit reduced myelination and decreased density of non-myelinated fibers

As Tau-null animals exhibit alteration in pain sensitivity, it was of great interest to monitor the structure and functionality of the sciatic nerve fibers that convey sensory input from the hind paw to the CNS. 
A

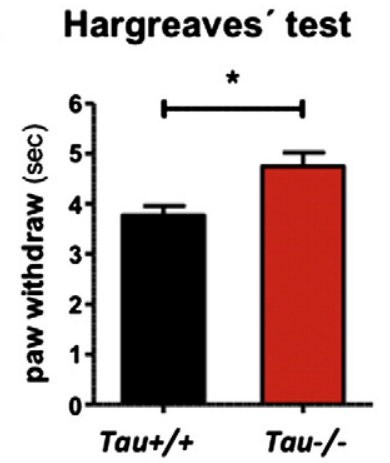

C

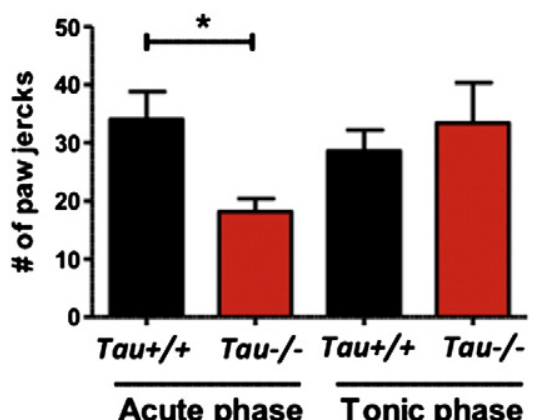

B

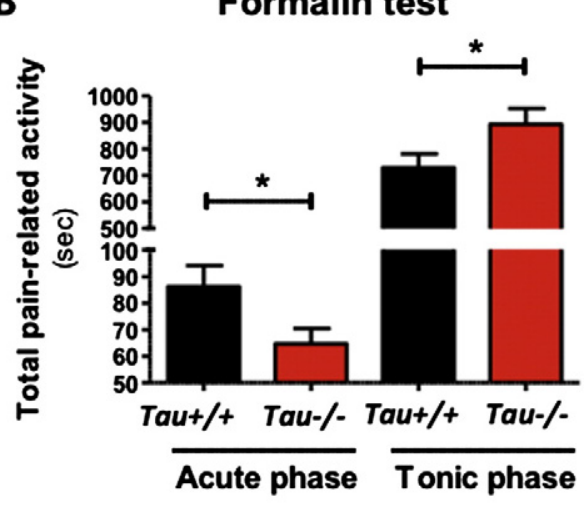

D

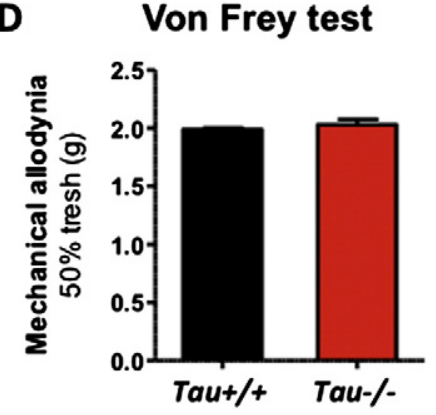

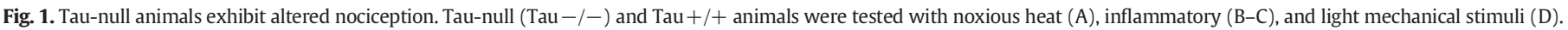

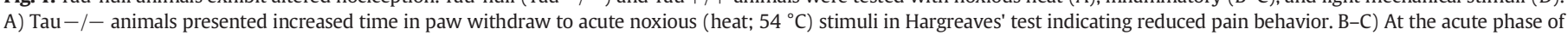

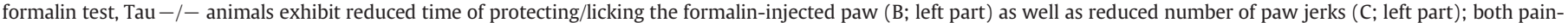

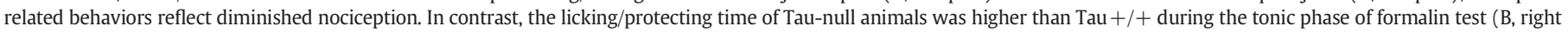
part). D) Tau-null and Tau +/+ animals presented no differences in the response to innocuous stimulus (light mechanical). Data presented as mean \pm SEM; ${ }^{*} \mathrm{p}<0.05$.

For that, we performed histological examinations of sciatic nerves using transmission electron microscopy (Fig. 3A). As shown in Fig. 3B, Taunull animals have reduced density of non-myelinated fibers (C-fibers) $(t=2.515, p<0.05)$, even though the number of non-myelinated fibers per Remak bundle was not affected (Fig. 3C). On the contrary, there was no difference in density of myelinated fibers between Tau-null and Tau $+/+$ animals as assessed by p-phenylene diamine (PPD) staining (Fig. 3D). Furthermore, we determined the g-ratio, an index of myelination independent of axonal diameter (Michailov et al., 2004), in sciatic nerves of animals of both genotypes (Fig. 4A). As shown in Fig. 4B, the mean g-ratio of Tau-null animals was significantly increased $(t=10.65, p<0.05)$ indicating thinner myelin sheaths in their sciatic nerve. Regarding the overall axonal population, the percentage of small-diameter myelinated axons (with diameter less than $4 \mu \mathrm{m}$ ) was significantly increased in Tau-null animals compared to Tau $+/+$ ones $(72.75 \% \pm 0.85$ and $65.40 \% \pm 2.50$, respectively; $\mathrm{t}=2.505, \mathrm{p}<0.05)$ indicating that the biggest difference in the individual g-ratios was found in the small-diameter fibers (Fig. 4C). Moreover, we monitored the levels of one of the main proteins found in compact myelin, the myelin basic protein (MBP) in sciatic nerves of Tau-null and Tau $+/+$ animals using western blot analysis. As shown at Fig. 4D, we found decreased levels of MBP protein in Tau-null animals, providing further support to the hypomyelination phenotype found by g-ratio analysis.

Sciatic nerve myelinated/unmyelinated CAPs ratio is reduced in Tau-null

The above morphological and molecular findings prompted us to monitor the functionality of sciatic nerve by measuring the conduction velocity of A- and C-fibers. For that purpose, Tau +/+ and Tau - / - sciatic nerves were repeatedly stimulated with current pulses of increasing amplitudes until saturation (Figs. 5A, B). As shown in Fig. 5C, no differences were found in the conduction velocity of Tau-null and Tau $+/+$ animals of both large-diameter myelinated (A-) and non-myelinated (C-) fibers. However, the ratio of the total area of the compound action potentials (CAPs) of myelinated vs unmyelinated fibers was significantly increased in Tau-null animals ( $t=2.367, p<0.05$; Fig. 5D) indicating an overall alteration in conduction properties of sciatic nerve of Tau-null animals.

\section{Discussion}

This study provides the first evidence that Tau depletion affects nociceptive behavior in mice and leads to morphofunctional alterations in primary afferent fibers. Our ultrastructural and biochemical analysis demonstrates a hypomyelination phenotype in sciatic nerves of Taunull animals. Indeed, a previous cell culture-based study by Klein et al. suggests the involvement of Tau protein in CNS myelin formation through its structural interaction with Fyn protein and microtubules (Klein et al., 2002). Transgenic loss of Fyn or its function is shown to result in severe hypomyelination (Sperber and McMorris, 2001; Umemori et al., 1994) but upstream partner of Fyn can also promote myelination independently of Fyn (Biffiger et al., 2000); the latter may explain why loss of Tau does not completely abrogate the myelination process in Tau-null mice. Supporting the involvement of Tau and microtubules in myelination process, the Taiep myelin mutant rats exhibit irregular microtubules, abnormal Tau accumulation and intracellular accumulation of myelin proteins in oligodendrocytes (Song et al., 2001), while PNS myelin-deficient mice exhibited reduction of Tau as well as other microtubule-associated proteins in their sciatic nerves (Kirkpatrick and Brady, 1994). A possible mechanism suggested by Song et al. focuses on the importance of Tau and microtubules in the transport of vesicles (Dixit et al., 2008) containing myelin components to the sheath formation of myelinating cells. On the other hand, as neuron-glial cell 


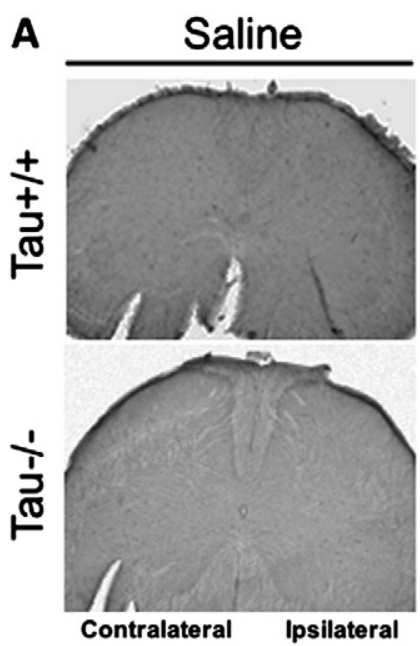

B

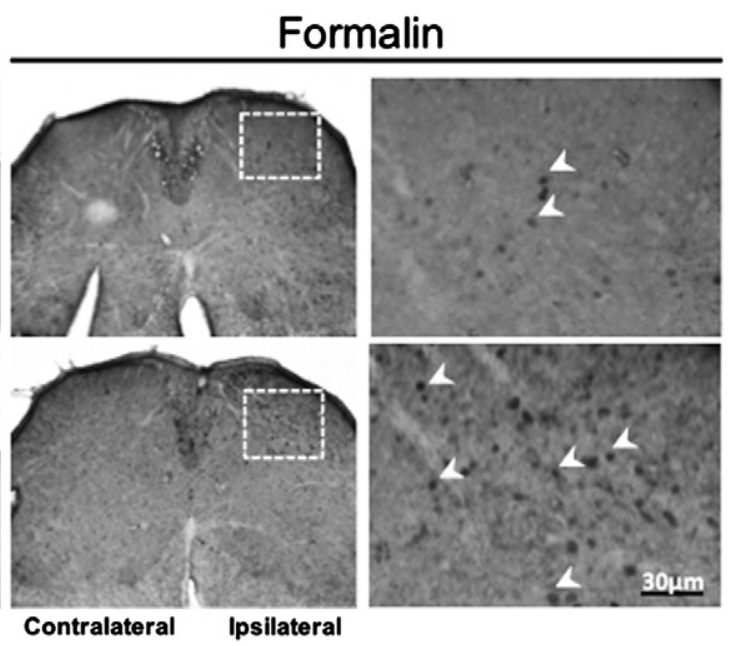

c-fos staining

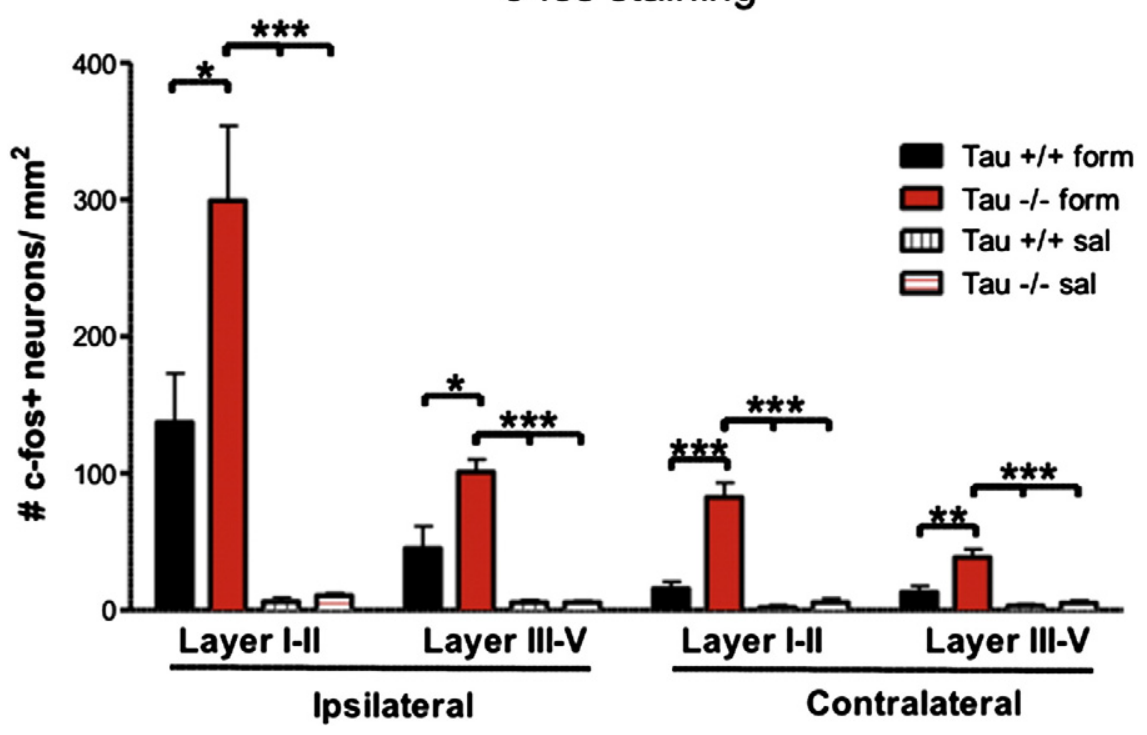

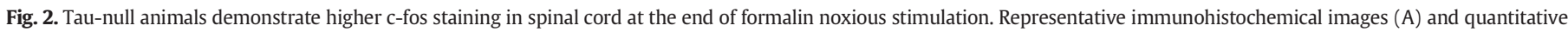

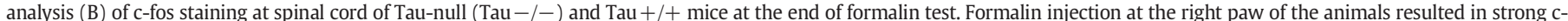

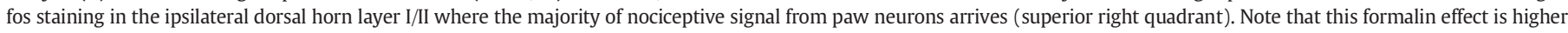

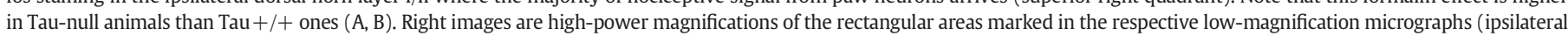

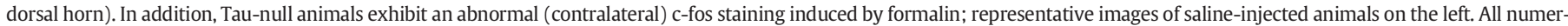
ical data shown represent mean \pm SEM. For individual comparisons, data were analyzed by one-way ANOVA followed by Tukey HSD post-hoc. ${ }^{*} \mathrm{p}<0.05,{ }^{* *} \mathrm{p}<0.01$ and ${ }^{* * *} \mathrm{p}<0.001$.

interaction(s) are essential for myelination, some studies showed that axonopathies are accompanied by myelin deficits (Wirths et al., 2006) raising the question whether axonal disturbances in neurons are preceded by the myelin deficits in myelinating (oligodendrocytes or Schwann) cells.

According to our observations, myelin alterations are restricted to small caliber axons $(<4 \mu \mathrm{m})$. Indeed, Tau protein is highly expressed in small-caliber axons of both adult CNS and PNS (low and high molecular weight Tau isoforms, respectively). Large-diameter axons on the other hand express very little amount of Tau (Nothias et al., 1995; Sato-Yoshitake et al., 1989). Accordingly, Harada and colleagues found that microtubule alterations were restricted to small-size axons in Tau-KO animals (Harada et al., 1994). This may result from the fact that, in contrast to large-caliber axons, Tau is the predominant MTassociated protein in small and medium caliber axons and thus, upon its depletion, Tau function in small-caliber axons cannot easily be compensated by other MT-associated proteins such as MAP1A, which is temporally increased in Tau-KO animals (Dawson et al., 2001; Harada et al., 1994).
Furthermore, we demonstrate a reduction in unmyelinated C-fiber density in sciatic nerve of Tau-null animals; importantly, unmyelinated C-fibers and small myelinated (A $\mathrm{A}$ ) fibers are the major systems conveying nociceptive inputs to the spinal cord although the participation of large myelinated $A \alpha / \beta$ fibers has also been demonstrated (Djouhri and Lawson, 2004; Todd, 2010). Tau depletion and the resulting loss of MT-Tau interaction may have a higher impact on C-fibers than on A-fibers as the MT/neurofilaments ratio in sciatic nerve is higher in unmyelinated fibers and thus, C-fibers may be structurally more dependent on these cytoskeleton elements (Friede and Samorajski, 1970). As a result, the overall ratio between unmyelinated/myelinated fibers was affected in Tau-null animals indicating a reorganization of the overall composition of the nerve fibers; this was in line with the electrophysiological monitoring of sciatic nerve functionality that shows an overall decrease in the proportion of total area of the compound action potentials (CAPs) of myelinated vs unmyelinated fibers. However, it is important to note that there were no major conduction velocity changes in both large myelinated and the remaining unmyelinated fibers suggesting that the existing fibers are properly conducting. 
A
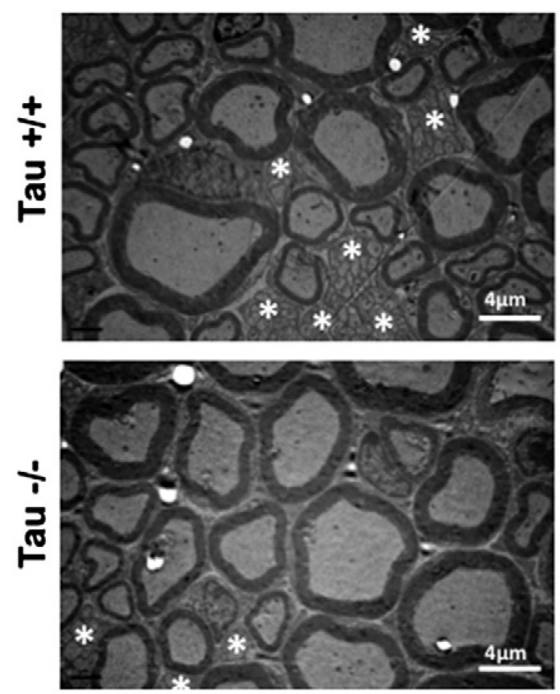

B

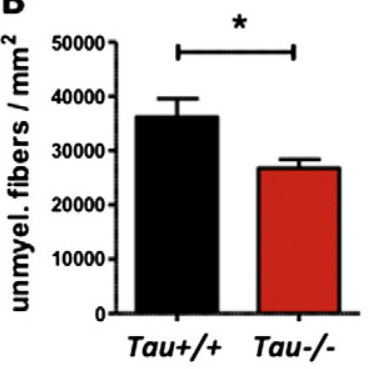

C

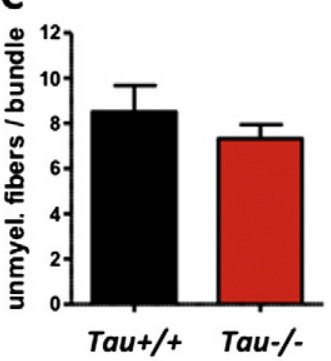

D

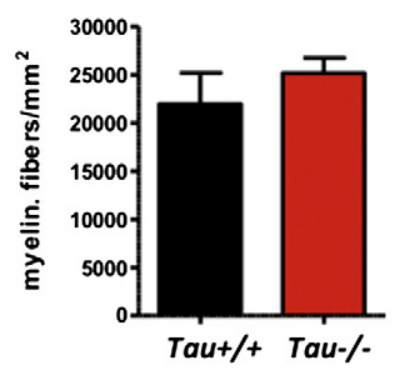

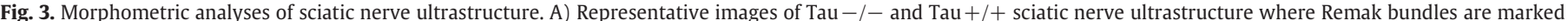

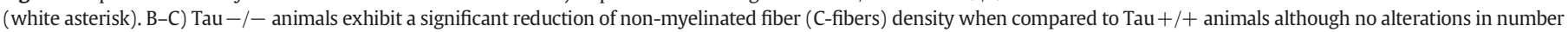

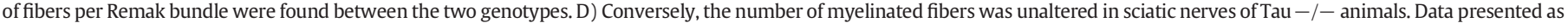
mean \pm SEM; ${ }^{*} \mathrm{p}<0.05$.

Tau-null animals demonstrated a decrease in pain sensitivity in the acute phase of the formalin test (first 5 min of noxious stimuli) that is in agreement with the results of the Hargreaves' test where a decrease in acute pain sensitivity in response to noxious (thermal) stimuli was also found. Although our pain-related behavioral readouts are motordependent, we can exclude potential confounders as no motor or
A

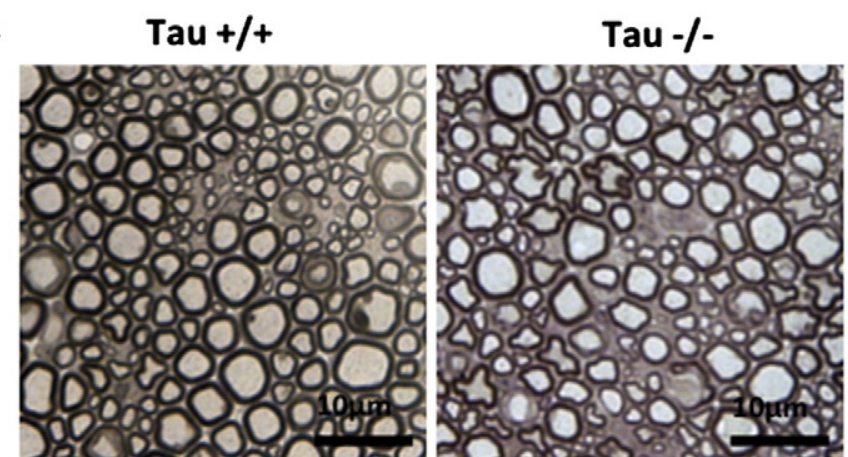

C

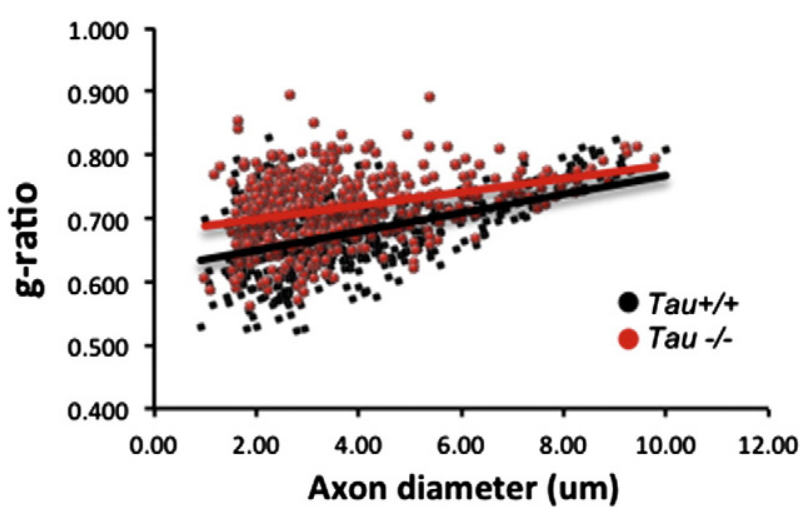

B

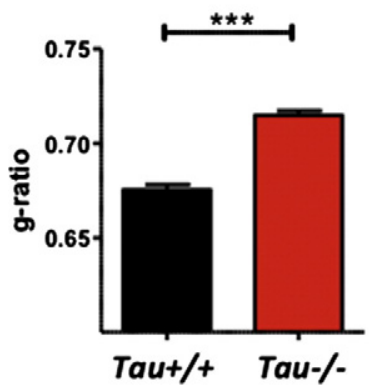

D
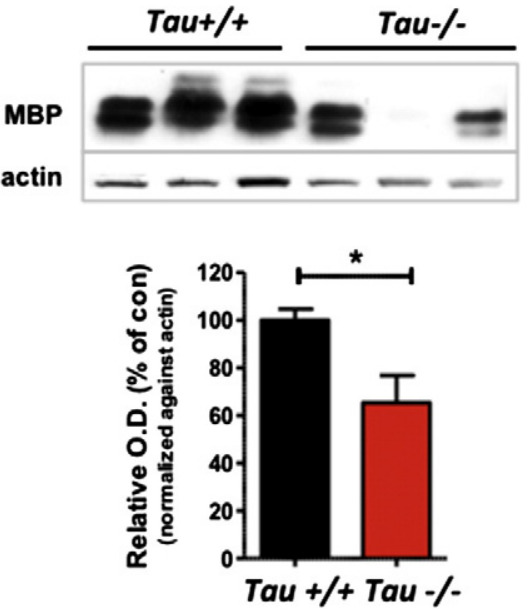

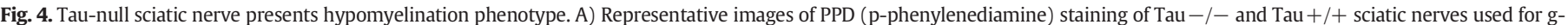

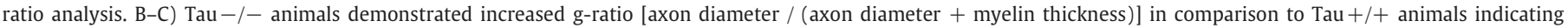

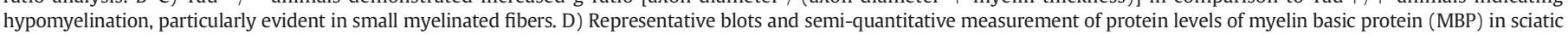
nerves assessed by WB analysis. Note the reduced MBP levels in sciatic nerves of Tau $-/-$ animals. Data presented as mean $\pm \mathrm{SEM}$; ${ }^{*} \mathrm{p}<0.05$. 
A

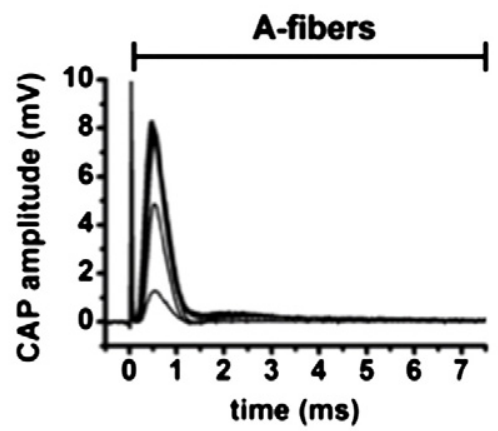

B

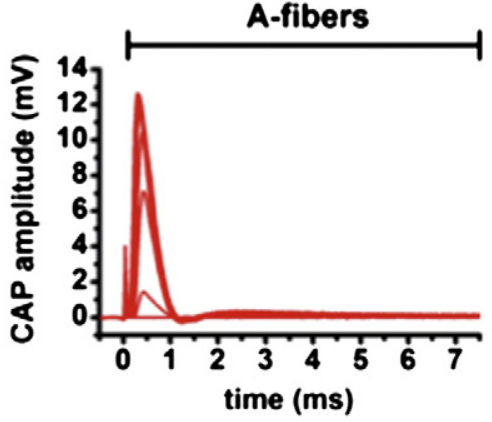

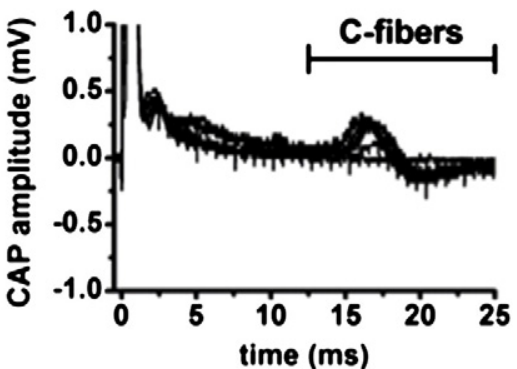

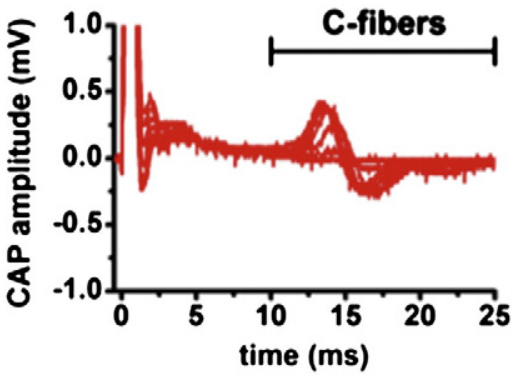

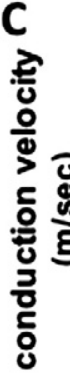

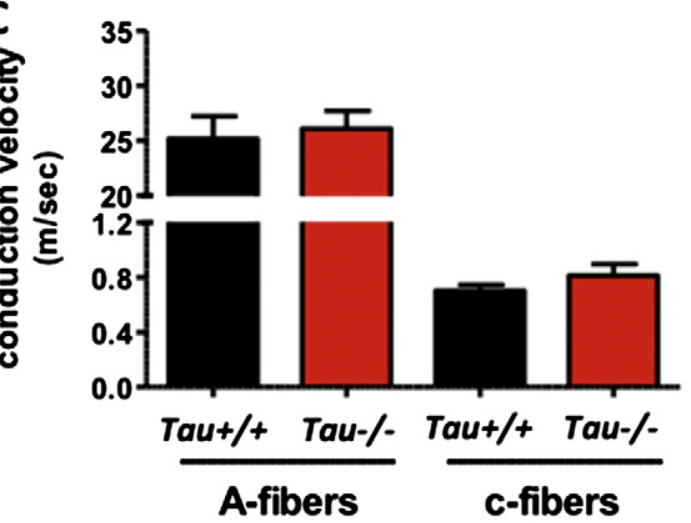

D

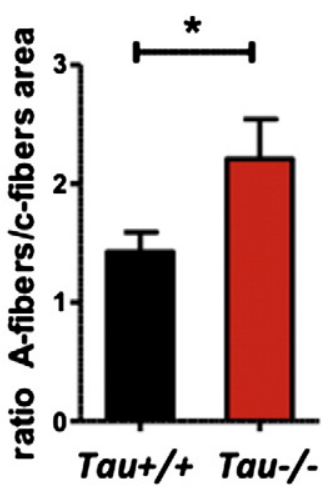

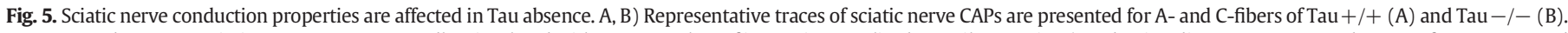

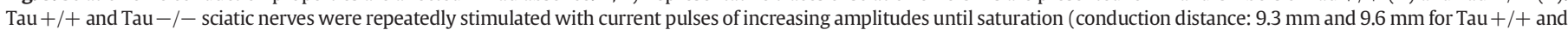

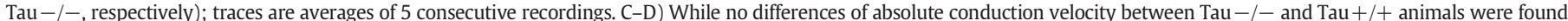

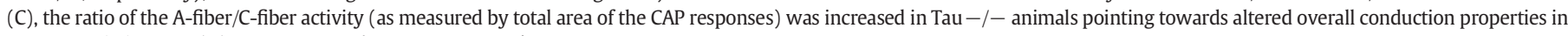
Tau $-/-$ sciatic nerve (D). Data presented as mean \pm SEM; ${ }^{*} \mathrm{p}<0.05$.

muscle strength deficits have been found in adult Tau-KO animals used in this study (Dawson et al., 2010; Gotz et al., 2013) while Tau-KO normal response to innocuous mechanical stimuli with reflex withdrawal (Von Frey) indicates that the acute pain (thermal) deficits assessed by Hargreaves' test were not due to a disruption of flexor motor neuron function. Thus, as acute pain is primarily dependent on primary afferent transmission, with limited intervention of central sensitization mechanisms (Liu et al., 2008), this decrease in acute pain is probably dependent on the reduction in unmyelinated C-fibers and the hypomyelination of A $\delta$-fibers. Previous studies reported a reduced response to neuropathy-triggered mechanical allodynia in animals lacking Fyn which exhibit a clear hypomyelination phenotype (Abe et al., 2005). The close interaction between Fyn and Tau (Klein et al., 2002) could explain the similarities between our and previous observations.

Contrary to the acute phase, the tonic phase of the formalin test is centrally mediated. In agreement, c-fos expression in the dorsal horn was augmented reflecting the increased pain-related behavior observed in Tau-null animals. The increased excitability of second order spinal cord nociceptive neurons, could be explained by a mechanism where a decrease in afferent inputs, due to a restrain in synaptic transmission
(D'Mello et al., 2011; Ittner and Gotz, 2011; Roberson et al., 2007), and in nociceptive conducting fibers, is compensated by an increase in somatic neuronal excitability to maintain a balanced spinal nociceptive circuit in Tau-null mice (Turrigiano, 2011). Alternatively, a decrease in spinal inhibition, caused by a decreased activity of the inhibitory circuit, could also account for such results (Zeilhofer et al., 2012). Overall, the centrally mediated hyperexcitability we described most probably also accounts for the contralateral c-fos expression observed in Tau-null mice. It is noteworthy that morphine-tolerant hyperalgesic animals exhibit a similar abnormal c-fos pattern in spinal cord (Rohde et al., 1997). Further analysis of the role of Tau on mechanisms of synaptic plasticity and neurotransmission at spinal cord is needed to clarify the above issue.

\section{Conclusions}

The present study provides the first in vivo evidence supporting the involvement of Tau protein in pain perception. This study suggests that, in the absence of Tau, two phenomena take place: i) nerve structural and functional alterations (e.g. reduced C-fiber density and A $\delta$-fiber 
hypomyelination) leading to decreased sensation to acute noxious stimuli and ii) increased excitability of second order spinal cord nociceptive neurons resulting in heightened pain-like behaviors in the tonic phase of formalin test. In the light of recent evidence suggesting novel roles for Tau at synaptic structure and function, future studies are necessary to clarify the mechanistic role of Tau in pain sensitization as this study suggests Tau-dependent alterations in the spinal nociceptive circuitry. Altogether, these findings are likely to be of relevance to understand the mechanisms underlying the clinical observations of myelin loss and diminished pain perception in AD patients (Bartzokis, 2011; Corbett et al., 2012).

\section{Conflict of interest statement}

Authors declare no conflicts of interest associated to this work.

\section{Acknowledgments}

We would like to thank Drs João Relvas, Joana Paes de Faria Monteiro and Nuno Dias for their comments in this work. Many thanks to Dr João Relvas for the MBP antibody. The work was supported by grants "SFRH/ BPD/80118/2011", “PTDC/SAU-NMC/113934/2009” funded by FCT Portuguese Foundation for Science and Technology and project DoIT Desenvolvimento e Operacionalização da Investigação de Translação (No. do projeto 13853), funded by Fundo Europeu de Desenvolvimento Regional (FEDER) through the Programa Operacional Fatores de Competitividade (POFC). Author's contributions: experimental design IS, HA, VP, AA, and NS; performed research - IS, HA, VP, AL, SL, SS, SP, AC, FPR, and RF; data analyses - IS, HA, AL, VP, SC, and FPR; and manuscript preparation - IS, HA, VP, and NS.

\section{References}

Abe, T., Matsumura, S., Katano, T., Mabuchi, T., Takagi, K., Xu, L., Yamamoto, A., Hattori, K., Yagi, T., Watanabe, M., Nakazawa, T., Yamamoto, T., Mishina, M., Nakai, Y.,Ito, S., 2005. Fyn kinase-mediated phosphorylation of NMDA receptor NR2B subunit at Tyr1472 is essential for maintenance of neuropathic pain. Eur. J. Neurosci. 22, 1445-1454

Almeida, A.,Storkson, R., Lima, D., Hole, K., Tjolsen, A., 1999. The medullary dorsal reticular nucleus facilitates pain behaviour induced by formalin in the rat. Eur. J. Neurosci. 11, $110-122$

Bartzokis, G., 2011. Alzheimer's disease as homeostatic responses to age-related myelin breakdown. Neurobiol. Aging 32, 1341-1371.

Biffiger, K., Bartsch, S., Montag, D., Aguzzi, A., Schachner, M., Bartsch, U., 2000. Severe hypomyelination of the murine CNS in the absence of myelin-associated glycoprotein and fyn tyrosine kinase. J. Neurosci. 20, 7430-7437.

Chaplan, S.R., Bach, F.W., Pogrel, J.W., Chung, J.M., Yaksh, T.L., 1994. Quantitative assessment of tactile allodynia in the rat paw. J. Neurosci. Methods 53,55-63.

Chen, S., Rio, C.,Ji, R.R., Dikkes, P., Coggeshall, R.E.,Woolf, C.J., Corfas, G., 2003. Disruption of ErbB receptor signaling in adult non-myelinating Schwann cells causes progressive sensory loss. Nat. Neurosci. 6, 1186-1193.

Corbett, A., Husebo, B., Malcangio, M., Staniland, A., Cohen-Mansfield, J., Aarsland, D., Ballard, C., 2012. Assessment and treatment of pain in people with dementia. Nat. Rev. Neurol. 8, 264-274.

Dawson, H.N., Ferreira, A., Eyster, M.V., Ghoshal, N., Binder, L.I.,Vitek, M.P., 2001. Inhibition of neuronal maturation in primary hippocampal neurons from tau deficient mice. J. Cell Sci. 114, 1179-1187

Dawson, H.N., Cantillana, V., Jansen, M., Wang, H., Vitek, M.P., Wilcock, D.M., Lynch, J.R., Laskowitz, D.T., 2010. Loss of tau elicits axonal degeneration in a mouse model of Alzheimer's disease. Neuroscience 169, 516-531.

Dixit, R., Ross, J.L., Goldman, Y.E.,Holzbaur, E.L., 2008. Differential regulation of dynein and kinesin motor proteins by tau. Science 319, 1086-1089.

Dixon, W.J., 1980. Efficient analysis of experimental observations. Annu. Rev. Pharmacol. Toxicol. 20, 441-462.
Djouhri, L.,Lawson, S.N., 2004. Abeta-fiber nociceptive primary afferent neurons: a review of incidence and properties in relation to other afferent A-fiber neurons in mammals. Brain Res. Brain Res. Rev. 46, 131-145.

D'Mello, R., Marchand, F., Pezet, S., McMahon, S.B., Dickenson, A.H., 2011. Perturbing PSD95 interactions with NR2B-subtype receptors attenuates spinal nociceptive plasticity and neuropathic pain. Mol. Ther. 19, 1780-1792.

Frandemiche, M.L., De Seranno, S., Rush, T., Borel, E., Elie, A., Arnal, I., Lante, F., Buisson, A., 2014. Activity-dependent tau protein translocation to excitatory synapse is disrupted by exposure to amyloid-beta oligomers. J. Neurosci. 34, 6084-6097.

Friede, R.L.,Samorajski, T., 1970. Axon caliber related to neurofilaments and microtubules in sciatic nerve fibers of rats and mice. Anat. Rec. 167, 379-387.

Gotz, J., Xia, D., Leinenga, G., Chew, Y.L., Nicholas, H., 2013. What renders TAU toxic. Front. Neurol. 4, 72 .

Harada, A., Oguchi, K., Okabe, S., Kuno, J., Terada, S., Ohshima, T.,Sato-Yoshitake, R.,Takei, Y., Noda, T.,Hirokawa, N., 1994. Altered microtubule organization in small-calibre axons of mice lacking tau protein. Nature 369, 488-491.

Ittner, L.M., Gotz, J., 2011. Amyloid-beta and tau-a toxic pas de deux in Alzheimer's disease. Nat. Rev. Neurosci. 12, 65-72

Jaggi, A.S., Singh, N., 2012. Mechanisms in cancer-chemotherapeutic drugs-induced peripheral neuropathy. Toxicology 291, 1-9.

Kirkpatrick, L.L., Brady, S.T., 1994. Modulation of the axonal microtubule cytoskeleton by myelinating Schwann cells. J. Neurosci. 14, 7440-7450.

Klein, C., Kramer, E.M., Cardine, A.M.,Schraven, B., Brandt, R., Trotter, J., 2002. Process outgrowth of oligodendrocytes is promoted by interaction of fyn kinase with the cytoskeletal protein tau. J. Neurosci. 22, 698-707.

Liu, X.J.,Gingrich, J.R.,Vargas-Caballero, M.,Dong, Y.N.,Sengar, A., Beggs, S.,Wang, S.H.,Ding, H.K.,Frankland, P.W.,Salter, M.W., 2008. Treatment of inflammatory and neuropathic pain by uncoupling Src from the NMDA receptor complex. Nat. Med. 14, 1325-1332.

Liu, Y.N., Yang, X.,Suo, Z.W., Xu, Y.M.,Hu, X.D., 2014. Fyn kinase-regulated NMDA receptor- and AMPA receptor-dependent pain sensitization in spinal dorsal horn of mice. Eur. J. Pain. http://dx.doi.org/10.1002/j.1532-2149.2014.00455.x (Electronic publication ahead of print).

Mandelkow, E.M., Mandelkow, E., 2012. Biochemistry and cell biology of tau protein in neurofibrillary degeneration. Cold Spring Harb. Perspect. Med. 2, a006247.

Michailov, G.V.,Sereda, M.W.,Brinkmann, B.G.,Fischer, T.M.,Haug, B.,Birchmeier, C., Role, L., Lai, C., Schwab, M.H., Nave, K.A., 2004. Axonal neuregulin-1 regulates myelin sheath thickness. Science 304, 700-703.

Nothias, F., Boyne, L., Murray, M., Tessler, A., Fischer, I., 1995. The expression and distribution of tau proteins and messenger RNA in rat dorsal root ganglion neurons during development and regeneration. Neuroscience 66, 707-719.

Ottoni, E.B., 2000. EthoLog 2.2: a tool for the transcription and timing of behavior observation sessions. Behav. Res. Methods Instrum. Comput. 32, 446-449.

Pinto, V., Derkach, V.A.,Safronov, B.V., 2008. Role of TTX-sensitive and TTX-resistant sodium channels in Adelta- and C-fiber conduction and synaptic transmission. J. Neurophysiol. 99, 617-628.

Pinto-Ribeiro, F., Amorim, D., David-Pereira, A., Monteiro, A.M., Costa, P., Pertovaara, A. Almeida, A., 2013. Pronociception from the dorsomedial nucleus of the hypothalamus is mediated by the rostral ventromedial medulla in healthy controls but is absent in arthritic animals. Brain. Res. Bull. 99C, 100-108.

Reichling, D.B.,Levine, J.D., 2011. Pain and death: neurodegenerative disease mechanisms in the nociceptor. Ann. Neurol. 69, 13-21.

Roberson, E.D., Scearce-Levie, K., Palop, J.J., Yan, F., Cheng, I.H., Wu, T., Gerstein, H., Yu, G.Q., Mucke, L., 2007. Reducing endogenous tau ameliorates amyloid beta-induced deficits in an Alzheimer's disease mouse model. Science 316, 750-754.

Rohde, D.S., Detweiler, D.J., Basbaum, A.I., 1997. Formalin-evoked Fos expression in spinal cord is enhanced in morphine-tolerant rats. Brain Res. 766, 93-100.

Sato-Yoshitake, R.,Shiomura, Y., Miyasaka, H.,Hirokawa, N., 1989. Microtubule-associated protein 1B: molecular structure, localization, and phosphorylation-dependent expression in developing neurons. Neuron 3, 229-238.

Song, J., Goetz, B.D., Kirvell, S.L., Butt, A.M., Duncan, I.D., 2001. Selective myelin defects in the anterior medullary velum of the taiep mutant rat. Glia 33,1-11.

Sperber, B.R., McMorris, F.A., 2001. Fyn tyrosine kinase regulates oligodendroglial cell development but is not required for morphological differentiation of oligodendrocytes. J. Neurosci. Res. 63, 303-312.

Todd, A.J., 2010. Neuronal circuitry for pain processing in the dorsal horn. Nat. Rev. Neurosci. 11, 823-836.

Turrigiano, G., 2011. Too many cooks? Intrinsic and synaptic homeostatic mechanisms in cortical circuit refinement. Annu. Rev. Neurosci. 34, 89-103.

Umemori, H.,Sato, S., Yagi, T.,Aizawa, S., Yamamoto, T., 1994. Initial events of myelination involve Fyn tyrosine kinase signalling. Nature 367, 572-576.

Wirths, O.,Weis, J.,Szczygielski, J.,Multhaup, G.,Bayer, T.A., 2006. Axonopathy in an APP/PS1 transgenic mouse model of Alzheimer's disease. Acta Neuropathol. 111, 312-319.

Zeilhofer, H.U.,Wildner, H., Yevenes, G.E., 2012. Fast synaptic inhibition in spinal sensory processing and pain control. Physiol. Rev. 92, 193-235. 